\title{
Existence of strong solutions for a class of semilinear evolution equations with nonlocal initial conditions
}

\author{
Pengyu Chen ${ }^{1}$, Yongxiang $\mathrm{Li}^{1 *}$ and Hongxia Fan ${ }^{1,2}$
}

\footnotetext{
* Correspondence: liyxnwnu@163. com

${ }^{1}$ Department of Mathematics, Northwest Normal University, Lanzhou 730070, People's Republic of China

Full list of author information is available at the end of the article
}

\begin{abstract}
This paper discusses the existence of strong solutions for a class of semilinear evolution equations with nonlocal initial conditions in Hilbert spaces. The discussion is based on analytic semigroups theory and fixed point theorem. An application to a partial differential equation with nonlocal condition is also considered. Mathematics Subject Classification(2010): 34G20; 34K30; 35D35; $47 D 06$.

Keywords: evolution equation, nonlocal initial condition, strong solution, analytic semigroups, existence
\end{abstract}

\section{Introduction}

In this paper, we discuss the existence of strong solutions for a class of semilinear evolution equations with nonlocal initial conditions in a Hilbert space $H$

$$
\begin{aligned}
& u^{\prime}(t)+A u(t)=f(t, u(t)), \quad t \in J, \\
& u(0)=\sum_{i=1}^{m} \gamma_{i} u\left(t_{i}\right),
\end{aligned}
$$

where $A: D(A) \subset H \rightarrow H$ is a positive definite self-adjoint operator, $f: J \times H \rightarrow H$ is given function satisfying some assumptions, $J$ denote the real compact interval $[0, a], a$ $>0$ is a constant, $0<t_{1}<t_{2}<\cdots<t_{m} \leq a, m \in \mathbb{N}, \gamma_{i}$ are real numbers, $\gamma_{i} \neq 0, i=1$, $2, \ldots, m$.

In 1990, Byszewski and Lakshmikantham [1] first investigated the nonlocal problems. They studied and obtained the existence and uniqueness of mild solutions for nonlocal differential equations. Since it is demonstrated that the nonlocal problems have better effects in applications than the classical Cauchy problems, differential equations with nonlocal conditions were studied by many authors and some basic results on nonlocal problems have been obtained, see [2-10] and the references therein. The importance of nonlocal conditions have also been discussed in [11-15]. For example, Deng [11] used the nonlocal condition of type (2) to describe the diffusion phenomenon of a small amount of gas in a transparent tube. In this case, condition (2) allows the additional measurements at $t_{i}, i=1,2, \ldots, m$, which is more precise than the measurement just at $t=0$. In [12], Byszewski pointed out that if $\gamma_{i} \neq 0, i=1,2, \ldots, m$, then the results can

(c) 2012 Chen et al; licensee Springer. This is an Open Access article distributed under the terms of the Creative Commons Attribution License (http://creativecommons.org/licenses/by/2.0), which permits unrestricted use, distribution, and reproduction in any medium, provided the original work is properly cited. 
be applied to kinematics to determine the location evolution $t \rightarrow u(t)$ of a physical object for which we do not know the positions $u(0), u\left(t_{1}\right), \ldots, u\left(t_{m}\right)$, but we know that the nonlocal condition (2) holds. Consequently, to describe some physical phenomena, the nonlocal condition can be more useful than the standard initial condition.

However, most of the existing articles only studied the existence and uniqueness of mild solutions for evolution equations with nonlocal conditions, there are very few papers considered the regularity results for nonlocal evolution equations. In $[2,3]$, Byszewski discussed the existence of strong and classical solutions for the evolution equation

$$
\frac{d u(t)}{d t}+A u(t)=f(t, u(t)), \quad t \in\left(t_{0}, t_{0}+a\right]
$$

with the nonlocal condition

$$
u\left(t_{0}\right)+g\left(t_{1}, t_{2}, \ldots, t_{m}, u(\cdot)\right)=u_{0}
$$

or

$$
u\left(t_{0}\right)+\sum_{i=1}^{m} \gamma_{i} u\left(t_{i}\right)=u_{0}
$$

in a reflexive Banach space $X$, but the conditions in [2,3] are very strong and some of them can not be satisfied in applications. In this paper, we obtained the existence of strong solutions for the nonlocal problem (1)-(2) in a frame of abstract Hilbert spaces. Furthermore, an optimal condition (see condition (H1)) on the coefficients $\gamma_{i}(i=1,2$, ..., $m$ ) to guarantee that the nonlocal problem (1)-(2) has solutions has been obtained. At last, we demonstrated that the abstract results obtained can be applied to the parabolic partial differential equation with nonlocal conditions. Our discussions are based on analytic semigroups theory and the famous Schauder's fixed point theorem.

\section{Preliminaries}

Let $H$ be a Hilbert space with inner product $(\cdot, \cdot)$, then $\|\cdot\|=\sqrt{(\cdot, \cdot)}$ is the norm on $H$ induced by inner product $(\cdot, \cdot)$. We denote by $C(J, H)$ the Banach space of all continuous $H$-value functions on interval $J$ with the maximum norm $\|u\|_{C}=\max _{t \in J}\|u(t)\|$ and by $\mathcal{L}(H)$ the Banach space of all linear and bounded operators on $H$.

Let $A: D(A) \subset H \rightarrow H$ be a positive definite self-adjoint operator in Hilbert space $H$ and it have compact resolvent. By the spectral resolution theorem of selfadjoint operator, the spectrum $\sigma(A)$ only consists of real eigenvalues and it can be arrayed in sequences as

$$
\lambda_{1}<\lambda_{2}<\cdots<\lambda_{n}<\cdots, \quad \lambda_{n} \rightarrow \infty(n \rightarrow \infty) .
$$

By the positive definite property of $A$, the first eigenvalue $\lambda_{1}>0$. From [16,17], we know that $-A$ generates an analytic operator semigroup $T(t)(t \geq 0)$ on $H$, which is exponentially stable and satisfies

$$
\|T(t)\| \leq e^{-\lambda_{1} t}, \quad \forall t \geq 0 .
$$

Since the positive definite self-adjoint operator $A$ has compact resolvent, the embed$\operatorname{ding} D(A) \otimes H$ is compact, and therefore $T(t)(t \geq 0)$ is also a compact semigroup. 
We recall some concepts and conclusions on the fractional powers of $A$. For $\alpha>0, A^{-\alpha}$ is defined by

$$
A^{-\alpha}=\frac{1}{\Gamma(\alpha)} \int_{0}^{\infty} s^{\alpha-1} T(s) d s,
$$

where $\Gamma(\cdot)$ is the Euler gamma function. $A^{-\alpha} \in \mathcal{L}(H)$ is injective, and $A^{\alpha}$ can be defined by $A^{\alpha}=\left(A^{-\alpha}\right)^{-1}$ with the domain $D\left(A^{\alpha}\right)=A^{-\alpha}(H)$. For $\alpha=0$, let $A^{\alpha}=I$.

We endow an inner product $(\cdot,)_{\alpha}=\left(A^{\alpha}\right.$., $A^{\alpha}$.) to $D\left(A^{\alpha}\right)$. Since $A^{\alpha}$ is a closed linear operator, it follows that $\left(D\left(A^{\alpha}\right),(\cdot,)_{\alpha}\right)$ is a Hilbert space. We denote by $H_{\alpha}$ the Hilbert space $\left(D\left(A^{\alpha}\right),(\cdot,)_{\alpha}\right)$. Especially, $H_{0}=H$ and $H_{1}=D(A)$. For $0 \leq \alpha<\beta, H_{\beta}$ is densely embedded into $H_{\alpha}$ and the embedding $H_{\beta} \otimes H_{\alpha}$ is compact. For the details of the properties of the fractional powers, we refer to [17] and [18].

It is well known [[16], Chapter 4, Theorem 2.9] that for any $u_{0} \in D(A)$ and $h \in C^{1}(J, H)$, the initial value problem of linear evolution equation (LIVP)

$$
\left\{\begin{array}{l}
u^{\prime}(t)+A u(t)=h(t), \quad t \in J, \\
u(0)=u_{0}
\end{array}\right.
$$

has a unique classical solution $u \in C^{1}(J, H) \cap C\left(J, H_{1}\right)$ expressed by

$$
u(t)=T(t) u_{0}+\int_{0}^{t} T(t-s) h(s) d s .
$$

If $u_{0} \in H$ and $h \in L^{1}(J, H)$, the function $u$ given by (8) belongs to $C(J, H)$, which is known as a mild solution of the LIVP(7). If a mild solution $u$ of the LIVP(7) belongs to $W^{1,1}(J, H) \cap L^{1}\left(J, H_{1}\right)$ and satisfies the equation for a.e. $t \in J$, we call it a strong solution.

Throughout this paper, we assume that

$$
\text { (H1) } \sum_{i=1}^{m}\left|\gamma_{i}\right|<e^{\lambda_{1} t_{1}}
$$

By assumption (H1), we have $\left\|\sum_{i=1}^{m} \gamma_{i} T\left(t_{i}\right)\right\| \leq \sum_{i=1}^{m}\left|\gamma_{i}\right| e^{-\lambda_{1} t_{1}}<1$. By operator spectrum theorem, we know that the operator

$$
\mathcal{B}:=\left(I-\sum_{i=1}^{m} \gamma_{l} T\left(t_{i}\right)\right)^{-1}
$$

exists, bounded and $D(\mathcal{B})=H$. Furthermore, by Neumann expression, $\mathcal{B}$ can be expressed by

$$
\mathcal{B}=\sum_{n=0}^{\infty}\left(\sum_{i=1}^{m} \gamma_{i} T\left(t_{i}\right)\right)^{n}
$$

Therefore

$$
\|\mathcal{B}\| \leq \sum_{n=0}^{\infty}\left\|\sum_{i=1}^{m} \gamma_{i} T\left(t_{i}\right)\right\|^{n}=\frac{1}{1-\left\|\sum_{i=1}^{m} \gamma_{i} T\left(t_{i}\right)\right\|} \leq \frac{1}{1-e^{-\lambda_{1} t_{1}} \sum_{i=1}^{m}\left|\gamma_{i}\right|} .
$$


To prove our main results, for any $h \in C(J, H)$, we consider the linear evolution equation nonlocal problem (LNP)

$$
\begin{aligned}
& u^{\prime}(t)+A u(t)=h(t), \quad t \in J, \\
& u(0)=\sum_{i=1}^{m} \gamma_{i} u\left(t_{i}\right) .
\end{aligned}
$$

Lemma 1 If the condition (H1) holds, then the LNP (12)-(13) has a unique mild solution $u \in C(J, H)$ given by

$$
u(t)=\sum_{i=1}^{m} \gamma_{i} T(t) \mathcal{B} \int_{0}^{t_{i}} T\left(t_{i}-s\right) h(s) d s+\int_{0}^{t} T(t-s) h(s) d s, \quad t \in J .
$$

Moreover, $u \in W^{1,2}(J, H) \cap L^{2}\left(J, H_{1}\right)$ is a strong solution of the LNP (12)-(13).

Proof. By (7) and (8), we know that Eq. (12) has a unique mild solution $u \in C(J, H)$ which can be expressed by

$$
u(t)=T(t) u(0)+\int_{0}^{t} T(t-s) h(s) d s .
$$

From (15),

$$
u\left(t_{i}\right)=T\left(t_{i}\right) u(0)+\int_{0}^{t_{i}} T\left(t_{i}-s\right) h(s) d s, \quad i=1,2, \ldots, m .
$$

By (13) and (16),

$$
u(0)=\sum_{i=1}^{m} \gamma_{i} T\left(t_{i}\right) u(0)+\sum_{i=1}^{m} \gamma_{i} \int_{0}^{t_{i}} T\left(t_{i}-s\right) h(s) d s .
$$

Since $I-\sum_{i=1}^{m} \gamma_{i} T\left(t_{i}\right)$ has a bounded inverse operator $\mathcal{B}$,

$$
u(0)=\sum_{i=1}^{m} \gamma_{i} \mathcal{B} \int_{0}^{t_{i}} T\left(t_{i}-s\right) h(s) d s .
$$

From (15) and (18), we know that $u$ satisfies (14).

Inversely, we can verify directly that the function $u \in C(J, H)$ given by (14) is a mild solution of the LNP (12)-(13).

By the maximal regularity of linear evolution equations with positive definite operator in Hilbert spaces (see [19], Chapter II, Theorem 3.3), when $u(0)=u_{0} \in H_{1 / 2}$, the mild solution of the LIVP (7) has the regularity

$$
u \in W^{1,2}(J, H) \cap L^{2}\left(J, H_{1}\right) \cap C\left(J, H_{1 / 2}\right)
$$

and it is a strong solution.

We note that $u(t)$ defined by (14) is the mild solution of the LIVP (7) for $u(0)=\sum_{i=1}^{m} \gamma_{i} \mathcal{B} \int_{0}^{t_{i}} T\left(t_{i}-s\right) h(s) d s$. By the representation (8) of mild solution, $u(t)=T$ $(t) u(0)+v(t)$, where $v(t)=\int_{0}^{t} T(t-s) h(s) d s$ Since the function $v(t)$ is a mild solution of the LIVP (7) with the null initial value $u(0)=\theta, v$ has the regularity (19). By the analytic property of the semigroup $T(t), T\left(t_{i}\right) u(0) \in D(A) H_{1 / 2}$. Hence, 
$u(0)=\sum_{i=1}^{m} \gamma_{i} T\left(t_{i}\right) u(0)+\sum_{i=1}^{m} \gamma_{i} v\left(t_{i}\right) \in H_{1 / 2}$. Using the regularity (19) again, we obtain that $u \in W^{1,2}(J, H) \cap L^{2}\left(J, H_{1}\right)$ and it is a strong solution of the LNP (12)-(13). $\square$

For any $r>0$, let

$$
\Omega_{r}=\{u \in C(J, H):\|u(t)\| \leq r, t \in J\},
$$

then $\Omega_{r}$ is a bounded closed and convex set on $C(J, H)$.

\section{Main results}

Theorem 1 Let $A$ be a positive definite self-adjoint operator in Hilbert space $H$ and it have compact resolvent, $f: J \times H \rightarrow H$ be continuous. If condition (H1) and the following condition

(H2) For some $r>0$, there exists a function $\phi \in L\left(J, \mathbb{R}^{+}\right)$such that for all $t \in J$ and $u$ $\in H$ satisfying $\|u\| \leq r,\|f(t, u)\| \leq \phi(t)$, hold, then the problem (1)-(2) has at least one strong solution $u \in W^{1,2}(J, H) \cap L^{2}\left(J, H_{1}\right)$.

Proof. We consider the operator $Q$ on $C(J, H)$ defined by

$$
\begin{aligned}
Q u(t)= & \sum_{i=1}^{m} \gamma_{i} T(t) \mathcal{B} \int_{0}^{t_{i}} T\left(t_{i}-s\right) f(s, u(s)) d s \\
& +\int_{0}^{t} T(t-s) f(s, u(s)) d s, \quad t \in J .
\end{aligned}
$$

By assumption (H1) and Lemma 1, it is easy to see that the mild solution of problem (1)-(2) is equivalent to the fixed point of the operator $Q$. In the following, we will prove that $Q$ has a fixed point by using the famous Schauder Fixed Point Theorem.

At first, we prove that $Q$ is continuous on $C(J, H)$. To this end, let $\left\{u_{n}\right\}_{n=1}^{\infty} \subset C(J, H)$ be a sequence such that $\lim _{n \rightarrow+\infty} u_{n}=u$ on $C(J, H)$. By the continuity of the nonlinear term $f$, for each $s \in J, \lim _{n \rightarrow+\infty} f\left(s, u_{n}(s)\right)=f(s, u(s))$. Therefore, we can conclude that

$$
\sup _{s \in J}\left\|f\left(s, u_{n}(s)\right)-f(s, u(s))\right\| \rightarrow 0, \quad \text { as } \quad n \rightarrow \infty .
$$

From (6) and (20), for $t \in J$, we have

$$
\begin{aligned}
& \left\|Q u_{n}(t)-Q u(t)\right\| \\
\leq & \frac{\sum_{i=1}^{m}\left|\gamma_{i}\right| e^{-\lambda_{1} t}}{1-e^{-\lambda_{1} t_{1}} \sum_{i=1}^{m}\left|\gamma_{i}\right|} \int_{0}^{t_{i}} e^{-\lambda_{1}\left(t_{i}-s\right)}\left\|f\left(s, u_{n}(s)\right)-f(s, u(s))\right\| d s \\
& +\int_{0}^{t} e^{-\lambda_{1}(t-s)}\left\|f\left(s, u_{n}(s)\right)-f(s, u(s))\right\| d s \\
< & \frac{e^{\lambda_{1} t_{1}}+1}{\lambda_{1}\left(1-e^{-\lambda_{1} t_{1}} \sum_{i=1}^{m}\left|\gamma_{i}\right|\right)} \sup _{s \in J}\left\|f\left(s, u_{n}(s)\right)-f(s, u(s))\right\|,
\end{aligned}
$$

which implies that

$$
\left\|Q u_{n}-Q_{u}\right\|_{C}<\frac{e^{\lambda_{1} t_{1}}+1}{\lambda_{1}\left(1-e^{-\lambda_{1} t_{1}} \sum_{i=1}^{m}\left|\gamma_{i}\right|\right)} \sup _{s \in J}\left\|f\left(s, u_{n}(s)\right)-f(s, u(s))\right\| .
$$


From (21), we know that

$$
\left\|Q u_{n}-Q u\right\|_{C} \rightarrow 0, \quad \text { as } n \rightarrow \infty .
$$

That is, $Q$ is continuous on $C(J, H)$.

Subsequently, we prove that $Q: C(J, H) \rightarrow C(J, H)$ is a compact operator. Let $0 \leq \alpha<\frac{1}{2}, 0<v<\frac{1}{2}-\alpha$. By [20], we can prove that the operator $Q$ defined by (20) maps $C(J, H)$ into $C^{v}\left(J, H_{\alpha}\right)$. By Arzela-Ascoli's theorem, the embedding $C^{v}\left(J, H_{\alpha}\right) \otimes C$ $(J, H)$ is compact. This implies that $Q: C(J, H) \rightarrow C(J, H)$ is a compact operator. Combining this with the continuity of $Q$ on $C(J, H)$, we know that $Q: C(J, H) \rightarrow C(J, H)$ is a completely continuous operator.

Next, we prove that there exists a positive constant $R$ big enough, such that $Q\left(\Omega_{R}\right) \subset$ $\Omega_{R}$. In fact, choosing

$$
R=\frac{\sum_{i=1}^{m}\left|\gamma_{i}\right|\left(1-e^{-\lambda_{1} t_{1}}\right)+1}{1-e^{-\lambda_{1} t_{1}} \sum_{i=1}^{m}\left|\gamma_{i}\right|} \int_{0}^{a} \varphi(s) d s .
$$

For any $u \in \Omega_{R}$, we have

$$
\begin{aligned}
\|Q u(t)\| \leq & \sum_{i=1}^{m}\left|\gamma_{i}\right| e^{-\lambda_{1} t}\|\mathcal{B}\| \int_{0}^{t_{i}} e^{-\lambda_{1}\left(t_{i}-s\right)}\|f(s, u(s))\| d s \\
& +\int_{0}^{t} e^{-\lambda_{1}(t-s)}\|f(s, u(s))\| d s \\
\leq & \frac{\sum_{i=1}^{m}\left|\gamma_{i}\right|}{1-e^{-\lambda_{1} t_{1}} \sum_{i=1}^{m}\left|\gamma_{i}\right|} \int_{0}^{t_{i}} \varphi(s) d s+\int_{0}^{t} \varphi(s) d s \\
\leq & \frac{\sum_{i=1}^{m}\left|\gamma_{i}\right|\left(1-e^{-\lambda_{1} t_{1}}\right)+1}{1-e^{-\lambda_{1} t_{1}} \sum_{i=1}^{m}\left|\gamma_{i}\right|} \int_{0}^{a} \varphi(s) d s \\
= & R .
\end{aligned}
$$

Therefore, $Q\left(\Omega_{R}\right) \subset \Omega_{R}$. Thus, $Q: \Omega_{R} \rightarrow \Omega_{R}$ is a completely continuous operator.

By Schauder Fixed Point Theorem, we know that $Q$ has at least one fixed point $u \in$ $\Omega_{R}$. Since $u$ is mild solution of the LNP (12)-(13) for $h(\cdot)=f(\cdot, u(\cdot))$, by Lemma $1, u \in$ $W^{1,2}(J, H) \cap L^{2}\left(J, H_{1}\right)$ is a strong solution of the problem (1)-(2).

If we replace the assumption (H2) by the following assumption (H2)* For some $r>0$, there exist a function $\phi \in L\left(J, \mathbb{R}^{+}\right)$and a non-decreasing continuous function $\psi: \mathbb{R}^{+} \rightarrow$ $\mathbb{R}^{+}$such that for all $t \in J$ and $u \in H$ satisfying $\|u\| \leq r$,

$$
\|f(t, u)\| \leq \varphi(t) \psi(\|u\|) .
$$

We have the following existence result.

Theorem 2 Let $A$ be a positive definite self-adjoint operator in Hilbert space $H$ and it have compact resolvent, $f: J \times H \rightarrow H$ be continuous. If the conditions (H1) and (H2)* are satisfied, then the problem (1)-(2) has at least one strong solution $u \in W^{1,2}(J, H) \cap$ 
$L^{2}\left(J, H_{1}\right)$ provided that there exists a constant $R$ with

$$
M \psi(R) \int_{0}^{a} \varphi(s) d s \leq R,
$$

where

$$
M=\frac{\sum_{i=1}^{m}\left|\gamma_{i}\right|\left(1-e^{-\lambda_{1} t_{1}}\right)+1}{1-e^{-\lambda_{1} t_{1}} \sum_{i=1}^{m}\left|\gamma_{i}\right|} .
$$

Proof. By the proof of Theorem 1, we know that the operator $Q: C(J, H) \rightarrow C(J, H)$ is completely continuous. For any $u \in \Omega_{R}$, from the assumption (H2)* and (22), we have

$$
\begin{aligned}
\|\mid Q u(t)\| \leq & \sum_{i=1}^{m}\left|\gamma_{i}\right| e^{-\lambda_{1} t}\|\mathcal{B}\| \int_{0}^{t_{i}} e^{-\lambda_{1}\left(t_{i}-s\right)} \| f(s, u(s) \| d s \\
& +\int_{0}^{t} e^{-\lambda_{1}(t-s)}\|f(s, u(s))\| d s \\
\leq & \frac{\sum_{i=1}^{m}\left|\gamma_{i}\right| \psi(R)}{1-e^{-\lambda_{1} t_{1}} \sum_{i=1}^{m}\left|\gamma_{i}\right|} \int_{0}^{t_{i}} \varphi(s) d s+\psi(R) \int_{0}^{t} \varphi(s) d s \\
\leq & \frac{\sum_{i=1}^{m}\left|\gamma_{i}\right|\left(1-e^{-\lambda_{1} t_{1}}\right)+1}{1-e^{-\lambda_{1} t_{1}} \sum_{i=1}^{m}\left|\gamma_{i}\right|} \psi(R) \int_{0}^{a} \varphi(s) d s \\
= & R,
\end{aligned}
$$

which implies $Q\left(\Omega_{R}\right) \subset \Omega_{R}$. Thus, $Q: \Omega_{R} \rightarrow \Omega_{R}$ is a completely continuous operator. By Schauder Fixed Point Theorem, we know that $Q$ has at least one fixed point $u \in \Omega$ ${ }_{R}$. Since $u$ is mild solution of the LNP (12)-(13) for $h(\cdot)=f(\cdot, u(\cdot))$, by Lemma $1, u \in W$ ${ }^{1,2}(J, H) \cap L^{2}\left(J, H_{1}\right)$ is a strong solution of the problem (1)-(2).

Corollary 1 Let $A$ be a positive definite self-adjoint operator in Hilbert space $H$ and it have compact resolvent, $f: J \times H \rightarrow H$ be continuous. If the conditions (H1) and (H2)* are satisfied, then the problem (1)-(2) has at least one strong solution $u \in W^{1,2}(J, H) \cap$ $L^{2}\left(J, H_{1}\right)$ provided that

$$
\liminf _{r \rightarrow+\infty} \frac{\psi(r)}{r}<\frac{1}{M \int_{0}^{a} \varphi(s) d s}
$$

where $M$ is defined by (23).

\section{An example}

In order to illustrate our main results, we consider the parabolic partial differential equation with nonlocal condition 


$$
\left\{\begin{array}{l}
\frac{\partial}{\partial t} u(x, t)-\frac{\partial^{2}}{\partial x^{2}} u(x, t)=f(x, t, u(x, t)), \quad(x, t) \in[0,1 \mid \times J \\
u(0, t)=u(1, t)=0, \quad t \in J \\
u(x, 0)=\sum_{i=1}^{m} \gamma_{i} u\left(x, t_{i}\right), \quad x \in[0,1]
\end{array}\right.
$$

where $J=[0, a], 0<t_{1}<t_{2}<\ldots<t_{m} \leq a, \gamma_{i}$ are real numbers, $\gamma_{i} \neq 0, i=1,2, \ldots, m, f$ : $[0,1] \times J \times \mathbb{R} \rightarrow \mathbb{R}$ is continuous.

Let $H=L^{2}(0,1 ; \mathbb{R})$ with the norm $\|\cdot\|_{2}$. We define the linear operator $A$ in Hilbert space $H$ by

$$
A u=-\frac{\partial^{2}}{\partial x^{2}} u, \quad u \in D(A)=H^{2}(0,1) \cap H_{0}^{1}(0,1),
$$

where $H^{2}(0,1)=W^{2,2}(0,1), H_{0}^{1}(0,1)=W_{0}^{1,2}(0,1)$. It is well know from [16,17] that $A$ is a positive definite self-adjoint operator on $H$ and $-A$ is the infinitesimal generator of an analytic, compact semigroup $T(t)(t \geq 0)$. Moreover, $A$ has discrete spectrum with eigenvalues $\lambda_{n}=n^{2} \pi^{2}, n \in \mathbb{N}$, associated normalized eigenvectors $v_{n}(x)=\sqrt{2} \sin n \pi x$, the set $\left\{v_{n}: n \in \mathbb{N}\right\}$ is an orthonormal basis of $H$ and

$$
T(t) u=\sum_{n=1}^{\infty} e^{-n^{2} \pi^{2} t}\left(u, v_{n}\right) v_{n}, \quad\|T(t)\| \leq e^{-\pi^{2} t}, \quad \forall t \geq 0 .
$$

Let $f(t, u(t))=f(\cdot, t, u(\cdot, t))$, then the problem (24) can be rewritten into the abstract form of problem (1)-(2).

Theorem 3 If the nonlinear term $f(x, t, u(x, t))=\sin u(x, t) /\left(t^{1 / 2}+1\right), x \in[0,1], t \in$ $J$ and $\sum_{i=1}^{m}\left|\gamma_{i}\right|<e^{\pi^{2}} t_{1}$, then the problem (24) has at least one strong solution $u \in C\left(J, H_{0}^{1}(0,1)\right) \cap L^{2}\left(J, H^{2}(0,1)\right) \cap W^{1,2}\left(J, L^{2}(0,1 ; \mathbb{R})\right)$.

Proof. Let $\phi(t)=t^{-1 / 2}$, from the condition $\sum_{i=1}^{m}\left|\gamma_{i}\right|<e^{\pi^{2}} t_{1}$, we easily see that the conditions (H1) and (H2) hold. Hence by Theorem 1, the problem (24) has a strong solution $u \in C\left(J, H_{0}^{1}(0,1)\right) \cap L^{2}\left(J, H^{2}(0,1)\right) \cap W^{1,2}\left(J, L^{2}(0,1 ; \mathbb{R})\right)$ in the sense of $L^{2}(0,1$; $\mathbb{R})$. $\square$

Theorem 4 If $\sum_{i=1}^{m}\left|\gamma_{i}\right|<e^{\pi^{2} t_{1}}, f:[0,1] \times J \times \mathbb{R} \rightarrow \mathbb{R}$ is continuous and satisfies the following conditions

(P1) For some $r>0$, there exists a function $\phi \in L\left(J, \mathbb{R}^{+}\right)$such that for all $t \in J, x \in[0$, 1] and $u \in \mathbb{R},|u| \leq r,|f(x, t, u(x, t))| \leq \phi(t)$,

(P2) There exists a function $c: \mathbb{R}^{+} \rightarrow \mathbb{R}^{+}$such that

$$
|f(x, t, \xi)-f(\gamma, s, \eta)| \leq c(r)\left(|x-\gamma|^{\mu}+|t-s|^{\mu / 2}+|\xi-\eta|\right)
$$

for any $r>0, \mu \in(0,1)$ and $(x, t, \xi),(y, s, \eta) \in[0,1] \times J \times[-r, r]$, then the problem (24) has at least one classical solution $u \in C^{2+\mu, 1+\mu / 2}([0,1] \times J)$.

Proof. From the condition $\sum_{i=1}^{m}\left|\gamma_{i}\right|<e^{\pi^{2} t_{1}}$ and assumption (P1), it is easy to verify that the conditions (H1) and (H2) are satisfied. Hence by Theorem 1, the problem (24) has at least one strong solution $u \in C\left(J, H_{0}^{1}(0,1)\right) \cap L^{2}\left(J, H^{2}(0,1)\right) \cap W^{1,2}\left(J, L^{2}(0,1 ; \mathbb{R})\right)$ in the sense of $L^{2}(0,1 ; \mathbb{R})$. Since the nonlinear term $f$ satisfies the condition (P2), by using the similar regularization method in [20], we can prove that $u \in C^{2+\mu, 1+\mu / 2}([0,1] \times J)$ is a classical solution of the problem (24). $\square$ 


\section{Acknowledgements}

This research was supported by NNSF of China (10871160) and NNSF of China (11061031).

\section{Author details}

'Department of Mathematics, Northwest Normal University, Lanzhou 730070, People's Republic of China 2Department of Mathematics, Lanzhou Jiaotong University, Lanzhou 730070, People's Republic of China

\section{Authors' contributions}

PC carried out the first draft of this manuscript, YL corrected this draft, HF prepared the final version of the manuscript. All authors read and approved the final version of the manuscript.

\section{Competing interests}

The authors declare that they have no competing interests.

Received: 6 April 2012 Accepted: 12 June 2012 Published: 12 June 2012

\section{References}

1. Byszewski, L, Lakshmikantham, V: Theorem about the existence and uniqueness of solutions of a nonlocal Cauchy problem in a Banach space. Appl Anal. 40, 11-19 (1990)

2. Byszewski, L: Theorems about the existence and uniqueness of solutions of a semilinear evolution nonlocal Cauchy problem. J Math Appl Anal. 162, 494-505 (1991). doi:10.1016/0022-247X(91)90164-U

3. Byszewski, L: Application of preperties of the right hand sides of evolution equations to an investigation of nonlocal evolution problems. Nonlinear Anal. 33, 413-426 (1998). doi:10.1016/50362-546X(97)00594-4

4. Lin, Y, Liu, JH: Semilinear integrodifferential equations with nonlocal Cauchy problem. Nonlinear Anal. 26, 1023-1033 (1996). doi:10.1016/0362-546X(94)00141-0

5. Benchohra, M, Ntouyas, SK: Existence of mild solutions of semilinear evolution inclusions with nonlocal conditions. Georgian Math J. 7, 221-230 (2000)

6. Wang, J, Zhou, $Y$, Wei, $W, X u, H$ : Nonlocal problems for fractional integrodifferential equations via fractional operators and optimal controls. Comput Math Appl. 62, 1427-1441 (2011). doi:10.1016/j.camwa.2011.02.040

7. Liang, J, Casteren, JV, Xiao, TJ: Nonlocal Cauchy problems for semilinear evolution equations. Nonlinear Anal. 50, 173-189 (2002)

8. Xiao, TJ, Liang, J: Existence of classical solutions to nonautonomous nonlocal parabolic problems. Nonlinear Anal. 63, 225-232 (2005). doi:10.1016/j.na.2005.05.008

9. Wang, J, Zhou, Y, Medved, M: Picard and weakly Picard operators technique for nonlinear differential equations in Banach spaces. J Math Anal Appl. 389, 261-274 (2012). doi:10.1016/j.jmaa.2011.11.059

10. Boucherif, A: Semilinear evolution inclutions with nonlocal conditions. Appl Math Lett. 22, 1145-1149 (2009). doi:10.1016/j.aml.2008.10.004

11. Deng, K: Exponential decay of solutions of semilinear parabolic equations with nonlocal initial conditions. J Math Anal Appl. 179, 630-637 (1993). doi:10.1006/jmaa.1993.1373

12. Byszewski, L: Existence and uniqueness of a classical solutions to a functional-differential abstract nonlocal Cauchy problem. J Math Appl Stoch Anal. 12, $91-97$ (1999). doi:10.1155/S1048953399000088

13. Benchohra, M, Ntouyas, SK: Nonlocal Cauchy problems for neutral functional differential and integrodifferential inclusions in Banach spaces. J Math Anal Appl. 258, 573-590 (2001). doi:10.1006/jmaa.2000.7394

14. Liang, J, Liu, JH, Xiao, TJ: Nonlocal Cauchy problems governed by compact operator families. Nonlinear Anal. 57, 183-189 (2004). doi:10.1016/.j.na.2004.02.007

15. Ezzinbi, K, Fu, X, Hilal, K: Existence and regularity in the $a$-norm for some neutral partial differential equations with nonlocal conditions. Nonlinear Anal. 67, 1613-1622 (2007). doi:10.1016/j.na.2006.08.003

16. Pazy, A: Semigroups of Linear Operators and Applications to Partial Differential Equations. Springer, Berlin (1983)

17. Henry, D: Geometric Theory of Semilinear Parabolic Equations. In Lecture Notes in Mathematics, vol. 840,Springer, New York (1981)

18. Xiang, $\mathrm{X}$, Ahmed, NU: Existence of periodic solutions of semilinear evilution equations with time lags. Nonlinear Anal. 18, 1063-1070 (1992). doi:10.1016/0362-546X(92)90195-K

19. Teman, R: Infinite-Dimensional Dynamical Systems in Mechanics and Physics. Springer, New York, 2 (1997)

20. Amann, H: Periodic solutions of semilinear parabolic equations. In: Cesari L, Kannan R, Weinberger R (eds.) Nonlinear Analysis: A Collection of Papers in Honor of Erich H Rothe. pp. 1-29. Academic Press, New York (1978)

doi:10.1186/1687-1847-2012-79

Cite this article as: Chen et al: Existence of strong solutions for a class of semilinear evolution equations with nonlocal initial conditions. Advances in Difference Equations 2012 2012:79. 